\title{
Upper extremity pain after spinal cord injury
}

\author{
M Dalyan*,1, DD Cardenas ${ }^{1}$ and B Gerard ${ }^{1}$ \\ ${ }^{1}$ Northwest Regional Spinal Cord Injury System, Department of Rehabilitation Medicine, University of Washington, \\ Seattle, Washington, USA
}

\begin{abstract}
Objective: Patients with spinal cord injury (SCI) may complain of upper extremity (UE) pain. The purpose of this study was to determine the frequency and severity of UE pain as well as its association with functional activities. Types of treatments that SCI patients received for UE pain and the benefits of these treatments were also identified.

Study design and methods: A questionnaire of demographic variables and measures of UE pain intensity, location, treatment, and interference with functional activities was mailed to 170 persons with SCI. Data was analyzed by descriptive and comparative statistics.

Results: Of the 130 persons who responded, $76(58.5 \%)$ (38 paraplegic, 38 tetraplegic patients) reported UE pain: $71 \%$ had shoulder pain, $53 \%$ wrist pain, $43 \%$ hand pain, and $35 \%$ elbow pain. Pain interfered with transfers in $65 \%(36 / 55)$ of the patients who were doing them. Of ten functional activities, pain was more likely to be associated with pressure reliefs, transfers, and wheelchair mobility. Sixty-three per cent sought medical treatment for pain, and of those, $90 \%$ received either physical therapy, pharmacological treatment or massage. Although only $27 \%$ had wheelchair or home modification or joint protection education, these approaches were helpful for almost all and very helpful or extremely helpful in $26.6 \%$ and $63.6 \%$ of the patients, respectively.

Conclusion: UE pain is a common problem in individuals with SCI and has impact on daily activities. UE pain prevention and management programs are needed for SCI patients.
\end{abstract}

Keywords: spinal cord injury; paraplegia; tetraplegia; pain; upper extremity pain

\section{Introduction}

Advances in the care of the individuals with spinal cord injury (SCI) have resulted in an increased life expectancy in this population. As an individual with SCI ages, an increase in the prevalence of problems like pain in the upper extremity (UE) might be expected. The structures of the UE are designed primarily for prehensile activities. Because the UEs of the SCI patient are also needed for daily functions such as mobility they are used more frequently and strenuously and subject to increased stresses compared to those of an able-bodied individual. In addition, because the SCI patients rely on their UEs more than able-bodied individuals, UE pain and impairment might be of greater functional consequence. In this study we aimed to determine the frequency and severity of UE pain according to specific UE regions, and its association with sex, age, time since SCI, age at the time of SCI, level of injury and completeness of injury. The impact of UE pain on functional activities and independence was also studied. Factors that led patients to seek diagnosis and treatment for UE pain, types of

*Correspondence: M Dalyan, MD, Turan Emeksiz Sokak, Kent Sitesi A Blok No: 5, 06700 Ankara, Turkey treatments they received and the benefits of these medical approaches were also identified.

\section{Methods}

Out-patients from the Northwest Regional SCI System $(n=170)$ who had been injured at least 1 year before this study were mailed a questionnaire which was specifically designed for this study. Demographic data were obtained. The patients were questioned about the time since SCI, injury level, completeness of the injury, onset time of UE pain after SCI, current activity levels and activity levels prior to the start of UE pain problem. The questions also dealt with the presence and severity of pain (mild, moderate, or severe) in the shoulders, elbows, wrists and hands. The limitation of independence because of UE pain was screened. Patients were asked to indicate how often (never/ rarely, sometimes/usually, or always) they had UE pain during functional daily activities. Patients also answered questions about their medical treatment seeking history for UE pain, types of treatments they received and benefits of these treatment approaches.

Data was analyzed by descriptive and comparative statistics. Comparative statistical analyses were per- 
formed by student's $t$-test and chi-square tests. Level of significance was set at $P<0.05$.

\section{Results}

Responses to the survey were received from $130(62$ patients with tetraplegia and 68 patients with paraplegia) of the 170 patients. The response rate was $76.5 \%$. Spinal cord injury database made it possible for us to analyze the differences between respondents and non-respondents regarding sex, ethnicity, and level of injury. These two groups differed only in the cause of injury and the difference could be accounted for primarily by the higher percentage of the nonrespondents who had gunshot wounds as a cause of injury. This finding is not surprising given that individuals who are subject to gunshot wounds are more likely to be in transient populations and less likely to be motivated to complete a survey.

Of the 130 subjects responding, 76 (58.5\%) (56 male, 20 female) had UE pain. The presence of UE pain by the SCI levels in our patients is shown in Table 1 . There were 38 tetraplegic and 38 paraplegic patients. The etiology of SCI was motor vehicle accident in $45 \%$ of the patients. UE pain began more than a year after SCI in $55.3 \%$ of the patients. Twenty-three per cent of the patients reported that UE pain started within a year after SCI and in $18.4 \%$ within a month.

There was a significant relationship between employment status and the presence of UE pain. More individuals with UE pain were found to be unemployed, and fewer individuals with UE pain were found to be employed full time. In the patient group with UE pain $20 \%$ were working full time and $21.4 \%$ were unemployed due to pain or illness but in the patient group without UE pain $45.2 \%$ were working full time and $7.1 \%$ were unemployed due to the illness $(P<0.01)$.

In the patient group with UE pain; average age was $42.2 \pm 12$ years, average age at the time of SCI was $29.6 \pm 13.2$ years and average time since injury was $11.8 \pm 8.5$ years. In the patient group without UE pain; average age was $47.7 \pm 15.9$ years, average age at the time of SCI was $32 \pm 18.9$ years the average time since injury was $12.2 \pm 10.1$ years. There were not any significant differences in age at the time of SCI and time since injury between the patients with and

Table 1 Levels of SCI in patients with UE pain $(n=76)$

\begin{tabular}{lrr}
\hline & $n$ & $\%$ \\
\hline C2-C4 & 11 & 14.5 \\
C5-C8 & 27 & 35.5 \\
T1-T5 & 6 & 7.9 \\
T6-T10 & 15 & 19.7 \\
T11-L2 & 16 & 21.1 \\
L3-S4/5 & 1 & 1.3 \\
\hline
\end{tabular}

without UE pain $(P>0.05)$. However, there was a significant difference between two groups on the basis of age $(P<0.01)$. This difference was largely accounted for by the greater proportion of individuals in the age group 31-40 with UE pain $(42 \%)$ compared to the lower proportion of the same age group in patients without UE pain $(24.5 \%)$. Further analyses were applied to screen the employment status in 31-40 age group and no significant differences were found when compared with the overall pain population.

In comparing groups with and without UE pain regarding sex, completeness of the spinal cord lesion, paraplegia versus tetraplegia; no significant differences were found between two groups $(P<0.05)$.

Table 2 shows the distribution and severity of UE pain by region. Shoulders were the most frequently reported region as painful $(71 \%$ of 76 persons with UE pain) followed by upper arm and wrist pain. Upper extremity pain was mild, moderate, or severe in $45 \%, 41 \%$ and $13 \%$ of the patients, respectively. There were no statistically significant differences neither in the occurrences nor in the severities of UE pain between right and left extremities in all regions. The current activity level of upper extremities was none to minimal in $17.1 \%$, moderate in $54 \%$ and heavy in $26.3 \%$ of the patients with UE pain.

Activity levels of upper extremities prior to the start of UE pain problem were none to minimal, moderate, or heavy in $23.7 \%, 30.3 \%$, and $42.1 \%$, respectively.

Sixty-five per cent $(36 / 55)$ of the patients who were doing transfers independently or with minimal help reported that pain interfered with transfers. Of ten functional activities pain was more likely to be associated with wheelchair mobility, ambulation, transfers, pressure relief and upper body dressing. Of 31 patients doing wheelchair sports, $24 \quad(77.4 \%)$ reported UE pain. Eighty per cent of the patients with UE pain reported interference of pain with sleep. Table 3 shows the impact of UE pain on functional activities revealed by the answers given to the applicable functional activity.

Twenty-six per cent $(20 / 76)$ of the patients with UE pain needed additional help for functional activities because of UE pain. Twenty-eight per cent (21/76) of the patients with UE pain reported limitation of

Table 2 Location and severity of UE pain in SCI patients $(n=76)$

\begin{tabular}{lcccc}
\hline & $\begin{array}{c}\text { Pain }+ \\
\mathrm{n}\end{array}$ & $\begin{array}{c}\text { Mild } \\
\%\end{array}$ & $\begin{array}{c}\text { Moderate } \\
\%\end{array}$ & $\begin{array}{c}\text { Severe } \\
\%\end{array}$ \\
\hline R shoulder & 51 & 35.3 & 45.1 & 19.6 \\
L shoulder & 54 & 42.6 & 46.3 & 11.1 \\
R elbow & 27 & 40.7 & 48.1 & 11.1 \\
L elbow & 21 & 42.9 & 42.9 & 14.3 \\
R wrist & 40 & 47.5 & 42.5 & 10 \\
L wrist & 34 & 61.8 & 29.4 & 8.8 \\
R hand & 32 & 46.8 & 40.6 & 12.5 \\
L hand & 33 & 48.4 & 36.4 & 15.2 \\
\hline
\end{tabular}


independence because of UE pain. There was a significant difference between those who reported the need for additional help in daily activities and those who did not in terms of severity of UE pain.

Sixty-three per cent $(48 / 76)$ of the patients sought medical treatment for upper extremity pain. Factors which we think that could effect seeking treatment were studied. Patients who sought treatment were significantly more likely to report higher severity levels of pain than those who did not seek treatment $(P<0.01)$. There was no clear association of seeking treatment with the number of painful regions in the upper extremity. There was a significant difference between those seeking treatment and those not seeking treatment in terms of impact on independence $(P<0.01)$ and in terms of needing additional help $(P<0.05)$. Thirty-three per cent of patients who sought treatment for UE pain reported needing additional help with daily activities whereas $14 \%$ of the patients not seeking medical help reported such a need. Eighty-nine per cent of the patients who did not seek treatment had no effect of UE pain on independence.

Physical therapy, medication, and massage were the most frequently received treatment modalities. Table 4 shows the types of treatments received and the benefits of them in SCI patients with UE pain. Although few patients received treatment approaches such as home modification, wheelchair modification or education in joint protection techniques, these were helpful for almost all and very helpful or extremely helpful in $26.6 \%$ and $63.6 \%$ of the patients, respectively.

\section{Discussion}

A considerable amount of interest has been directed toward the musculoskeletal problems of patients with chronic SCI. The prevalence of UE pain in SCI patients following comprehensive rehabilitation has been thoroughly studied. Most of these studies dealt with the presence of shoulder or wrist pain and carpal tunnel syndrome. ${ }^{1-4}$ However, severity of UE pain, functional limitations and the social impact of this type of pain have not been studied sufficiently and little is known about the treatment and outcome of UE pain problems in individuals with SCI.

Nichols found shoulder pain in $51 \%$ of SCI patients. ${ }^{1}$ Bayley reported that the prevalence of shoulder pain in SCI patients was $30 \%$ and the most common diagnosis was chronic impingement. ${ }^{5}$ Both attributed shoulder problems to overuse. In contrast another study found that moderate joint activity

Table 3 Impact of UE pain on functional activities in SCI patients

\begin{tabular}{|c|c|c|c|c|}
\hline & $\begin{array}{c}\text { Applicable } \\
\mathrm{n}\end{array}$ & $\begin{array}{c}\text { Never or rarely } \\
\%\end{array}$ & $\begin{array}{c}\text { Sometimes or usually } \\
\%\end{array}$ & $\begin{array}{c}\text { Always } \\
\%\end{array}$ \\
\hline Wheelchair mobility (electric or manual) & 64 & 10.9 & 78.1 & 10.9 \\
\hline Ambulation & 32 & 15.6 & 59.4 & 15.6 \\
\hline Transfers & 58 & 25.9 & 58.6 & 15.5 \\
\hline Pressure relief & 56 & 35.7 & 44.6 & 19.6 \\
\hline Upper body dressing & 64 & 35.9 & 59.4 & 4.7 \\
\hline Lower body dressing & 56 & 41.1 & 46.4 & 10.7 \\
\hline Grooming & 65 & 50.8 & 40 & 7.7 \\
\hline Feeding & 64 & 54.7 & 39.1 & 4.7 \\
\hline Wheelchair sports & 31 & 19.4 & 61.3 & 16.1 \\
\hline Employment (full time, part time, student) & 48 & 20.8 & 72.9 & 6.25 \\
\hline Sleeping or at bed time & 76 & 18.4 & 59.2 & 21.1 \\
\hline
\end{tabular}

Table 4 Types and effects of treatments received for UE pain in SCI patients $(n=48)$

\begin{tabular}{|c|c|c|c|c|}
\hline & $\begin{array}{c}\text { Treatment } \\
\mathrm{n}\end{array}$ & $\begin{array}{c}\text { Not helpful } \\
\%\end{array}$ & $\begin{array}{c}\text { Helpful } \\
\%\end{array}$ & $\begin{array}{c}\text { Very helpful } \\
\text { |extremely helpful } \\
\%\end{array}$ \\
\hline Physical therapy & 41 & 17.1 & 56.1 & 24.4 \\
\hline Medication & 46 & 10.9 & 52.2 & 32.6 \\
\hline Massage & 42 & 7.1 & 40.5 & 47.6 \\
\hline Acupuncture & 7 & 57.1 & 28.6 & 14.3 \\
\hline Surgery & 3 & 0 & 66.7 & 33.3 \\
\hline Home modification & 11 & 0 & 27.3 & 63.6 \\
\hline Wheelchair modification & 11 & 9.1 & 27.3 & 54.5 \\
\hline Education in joint protection techniques & 15 & 13.3 & 53.3 & 26.6 \\
\hline
\end{tabular}


protected shoulders from degeneration; radiographs and medical records of 38 patients with paraplegia 20 years or more postinjury were studied, and it was found that $45 \%$ of 'inactive' patients had degenerative changes compared to $18 \%$ in the 'active' group. ${ }^{6}$ Patients utilizing crutches for ambulation were evaluated and it was reported that there was no clinical or radiological evidence of degeneration in the shoulder, but subjects complained of wrist, hand, neck and shoulder soreness after ambulation. ${ }^{7}$ Sie et al examined 239 SCI patients and reported that $55 \%$ of the patients with tetraplegia had UE pain; most commonly at the shoulder and $64 \%$ of the patients with paraplegia had UE pain; complaints related to carpal tunnel syndrome were the most common, followed by those related to shoulder pain. ${ }^{3} \mathrm{~A}$ survey of 800 SCI patients concluded that $72.7 \%$ of the respondents $(66 \%)$ reported some degree of chronic pain at the wrist and shoulder. ${ }^{8}$ Wheelchair propulsion and transfers caused most pain. Patient's age, neurologic level and time since injury were not statistically significant in the study and various routine therapies were not effective.

A decrease in the prevalence of UE pain in tetraplegic patients after the first 5 years post-injury was noted. ${ }^{3,9}$ Similarly, the prevalence of all UE pain in patients with paraplegia more than 20 years postinjury was reported to be low. ${ }^{3}$

In our study, $58.5 \%$ of SCI patients reported UE pain. The most common painful regions were shoulders followed by wrists. Upper extremity pain was mild, moderate or severe in $45 \%, 41 \%$ and $13 \%$ of the patients, respectively. Time since injury was not significant but patients in the $31-40$ age group tended to have an incidence of UE pain more than the other age groups, a finding which we believe is associated with more activity and resulting overuse in this rather younger age group. Spinal cord injured patients at even relatively young ages should be watched closely for the development of overuse related problems.

Bayley studied intra-articular pressure in paraplegic patients with shoulder pain during different activities including transfers and found that it was 2.5 times above the arterial pressure and postulated that increase in pressure leads to degenerative changes in the shoulder joint complex. ${ }^{5}$ Gellman et al reported $68 \%$ incidence of UE pain in 84 patients with paraplegia and a $30 \%$ incidence of shoulder pain during transfer activities. ${ }^{10}$ Upper extremity pain in our patients interfered mostly with wheelchair mobility, ambulation and transfers: activities that require loading and repetitive movements on the shoulders and wrists. Additionally, a significant number of our patients reported that they had UE pain during pressure relief and these patients might sometimes be avoiding this very important preventive measure and even cause pressure ulcers.

The impact of UE pain on daily activities of SCI patients might increase the disability from SCI. Pentland reported that although $60 \%$ of paraplegics had upper limb pain during some aspect of daily activities performance; $29 \%$ had made changes to deal with this, and $11 \%$ sought assistance with functional activities due to upper limb pain. ${ }^{11}$ Twenty-eight per cent of SCI patients with UE pain in this study had limitation of independence and $26 \%$ needed additional help to perform functional activities due to this problem and the severity of pain contributed significantly to the loss of independence. Patients in Pentland's study could have less severe pain and there could be psychosocial differences when compared with our study group as more patients with UE pain in our study reported loss of independence due to pain in their upper extremities. We analyzed the relationship between the employment status and the presence of UE pain which has not been studied before. Our results showed that more individuals with UE pain were unemployed when compared with the persons without UE pain. The UE pain itself might have prevented employment or the pain was worse in those unemployed because of a lack of distraction or because of an element of depression. We were not able to answer these questions within the limitations of a survey type of study but we think that preventative and management steps of musculoskeletal problems are required to ensure continued independence, productivity, and quality of life in SCI patients over time.

We think that most of the SCI patients perceive the presence of musculoskeletal pain significant as our results indicated that more than half of these individuals $(63 \%)$ sought medical treatment. But the patients tended to seek diagnosis and treatment when the pain was severe and caused loss of independence. Given the high incidence and the related outcomes of upper limb pain in the SCI population, there is a remarkable lack of data about the treatment of such problems. In the present study, medical approaches for the treatment of UE pain mostly consisted of physical therapy, medication and massage and the effects were not permanently satisfactory. In a recent case series study, it was shown that surgical management of rotator cuff tears in paraplegics were not always successful and the need for conservative management for both the involved and uninvolved shoulders were emphasized. ${ }^{12}$ We found that although the approaches like wheelchair modification, home modification, and education in joint protection techniques were prescribed less than the traditional modalities they were very helpful in most of the patients.

We conclude that there is a need for the implementation of upper limb pain prevention and management programs for persons with SCI both in the early phases of a rehabilitation program and during ongoing care even for decades. These programs should include patient education about the basic biomechanical principles on avoiding impingement and overuse, eliminating the damaging patterns, managing the early signs of strain and overuse and knowledge of several alternative techniques of daily 
activities. Education and training in endurance and balanced strengthening of muscles acting around the shoulder and optimizing posture to achieve a normal alignment of shoulder, head, and the spine are critical for the avoidance of injuries. Ergonomically designed environmental changes and wheelchair, home and work modifications should always be considered.

Further clinical and biomechanical research needs to be done to improve the preventive measures and treatment methods of UE pain in SCI patients in order for them to maintain optimal functional status.

\section{Acknowledgements}

This study was supported in part by the National Institute on Disability and Rehabilitation Research (NIDRR).

\section{References}

1 Nichols PJR, Norman PA, Ennis JR. Wheelchair user's shoulder? Shoulder pain in patients with spinal cord lesions. Scand J Rehab Med 1979; 11: 29-32.

2 Campbell CC, Koris MJ. Etiologies of shoulder pain in cervical spinal cord injury. Clin Orthop 1996; 322: 140-145.
3 Sie IH, Waters RL, Adkins RH, Gelmann H. Upper extremity pain in the postrehabilitation spinal cord injured patient. Arch Phys Med Rehabil 1992; 73: 44-48.

4 Alijure $\mathbf{J}$ et al. Carpal tunnel syndrome in paraplegic patients. Paraplegia 1985; 23: $182-186$

5 Bayley JC, Cochran TP, Sledge CB. The weight bearing shoulder. The impingement syndrome in paraplegics. J Bone Joint Surg [Am] 1987; 69: 676-678.

6 Wylie EJ, Chakera TMH. Degenerative joint abnormalities in patients with paraplegia duration greater than 20 years. Paraplegia 1988; 26: $101-106$.

7 Wing PC, Tredwell SJ. The weight bearing shoulder. Paraplegia 1983; 21: $107-113$.

8 Subbarao JV, Klopfstein J, Turpin R. Prevalence and impact of wrist and shoulder pain in patients with spinal cord injury. $J$ Spinal Cord Med 1995; 18: 9-13.

9 Silfverskiold J, Waters RL. Shoulder pain and functional disability in spinal cord injury patients. Clin Orthop Related Res 1991; 272: $141-145$.

10 Gelmann H, Sie I, Waters RL. Late complications of the weightbearing upper extremity in paraplegic patient. Clin Orthop Related Res 1988; 233: $132-135$.

11 Pentland WE, Twomey LT. Upper limb function in persons with long term paraplegia and implications for independence: Part I. Paraplegia 1994; 32: $211-218$.

12 Goldstein B, Young J, Escobedo EM. Rotator cuff repairs in individuals with paraplegia. Am J Phys Med Rehabil 1997; 76 $316-322$ 